\title{
Nonlinear couplers based on plasmonic waveguides
}

\section{Acopladores no lineales con guías plasmónicas}

\author{
José Ramón Salgueiro ${ }^{(1, *)}$, Yuri S. Kivshar(2) \\ 1. Departamento de Física Aplicada, Universidade de Vigo, As Lagoas s/n, 32004 Ourense, Spain. \\ 2. Nonlinear Physics Center, Research School of Physics and Engineering, Australian National University, Canberra \\ ACT 0200, Australia. \\ (*) Email: jrs@uvigo.es \\ Recibido / Received: 07/10/2011. Aceptado / Accepted: 03/01/2011.
}

\begin{abstract}
:
In this work we study directional couplers made of waveguides with metallic claddings and nonlinear dielectric cores. The nonlinear modes are calculated describing the dependence of their shape with the increasing power. Using FDTD simulations we describe the switching properties, studying the power transfer between the waveguides and evaluating the effect of optical losses. We propose and analyse the use of tapered waveguides to compensate losses and improve the efficiency of the device.
\end{abstract}

Key words: Plasmonics, Directional Couplers, Switching, Tapers, Nonlinear Guides.

\section{RESUMEN:}

En este trabajo se estudian acopladores direccionales formados con guías que presentan cubiertas metálicas y núcleos dieléctricos no lineales. Se calculan los modos no lineales del acoplador describiendo su forma en función de la potencia. Por medio de simulaciones FDTD se analiza su uso para operaciones de conmutación óptica, estudiando la transferencia de potencia entre las guías y evaluando el efecto de las pérdidas. Se propone y analiza el uso de guías en forma de taper con el objeto de compensar las pérdidas y mejorar la eficiencia del dispositivo.

Palabras clave: Plasmónica, Acopladores Direccionales, Conmutación, Tapers, Guías no Lineales.

\section{REFERENCIAS Y ENLACES / REFERENCES AND LINKS}

[1]. S. A. Maier, Plasmonics: Fundamentals and Applications, Springer, New York (2007).

[2]. S. I. Bolzhevolnyi, V. S. Volkov, E. Devaux, J. Y. Laluet, T. W. Ebbesen, "Channel plasmon subwavelength waveguide components including interferometers and ring resonators", Nature 440, 508-511 (2006).

[3]. R. Yang, M. A. G. Abushagur, Z. Lu, "Efficiently squeezing near infrared light into a $21 \mathrm{~nm}$-by-24 nm nanospot", Opt. Express 16, 20142-20148 (2008).

[4]. D. K. Gramotnev, K. C. Vernon, D. F. P. Pile, "Directional coupler using gap plasmon waveguides", Appl. Phys. B 93, 99-106 (2008).

[5]. Z. Chen, T. Holmgaard, S. I. Bozhevolnyi, A. V. Krasavin, A. V. Zayats, L. Markey, A. Dereux, "Wavelength-selective directional coupling with dielectric-loaded plasmonic waveguides", Opt. Lett. 34, 810-812 (2009).

[6]. T. Holmgaard, Z. Chen, S. I. Bozhevolnyi, L. Markey, A. Dereux, "Design and characterization of dielectric-loaded plasmonic directional couplers", J. Lightwave Technol. 27, 5521-5528 (2009).

[7]. A. Degiron, S. Y. Cho, T. Tyler, N. M. Jokerst, D. R. Smith, "Directional coupling between dielectric and long-range plasmon waveguides", New J. Phys. 11, 015002 (2009).

[8]. V. M. Agranovich, V. S. Babichenko, V. Y. Chernyak, "Nonlinear surface polaritons", JETP Lett. 32, 512515 (1980). 
[9]. G. I. Stegeman, C. T. Seaton, J. Ariyasu, R. F. Wallis, A. A. Maradudin, "Nonlinear electromagnetic waves guided by a single interface", J. Appl. Phys. 58, 2453-2459 (1985).

[10]. D. Mihalache, G. I. Stegeman, C. T. Seaton, E. M. Wright, R. Zanoni, A. D. Boardman, T. Twardowski, "Exact dispersion relations for transverse magnetic polarized guided waves at a nonlinear interface", Opt. Lett. 12, 187-189 (1987).

[11]. A. R. Davoyan, I. V. Shadrivov, Y. S. Kivshar, "Nonlinear plasmonic slot waveguides", Opt. Express 16, 21209-21214 (2008).

[12]. J. R. Salgueiro, Y. S. Kivshar, "Nonlinear plasmonic directional couplers", Appl. Phys. Lett. 97, 081106 (2010).

[13]. A. R. Davoyan, I. V. Shadrivov, A. A. Zharov, D. K. Gramotnev, Y. S. Kivshar, "Nonlinear nanofocusing in tapered plasmonic waveguides", Phys. Rev. Lett. 105, 116804 (2010).

[14]. A. R. Davoyan, I. V. Shadrivov, Y. S. Kivshar, D. K. Gramotnev, “Optimal tapers for compensating losses in plasmonic waveguides”, Phys. Status Solidi-R 4, 277-279 (2010).

[15]. D. F. P. Pile, D. K. Gramotnev, "Adiabatic and nonadiabatic nanofocusing of plasmons by tapered gap plasmon waveguides”, Appl. Phys. Lett. 89, 041111 (2006).

[16].S. I. Bozhevolnyi, K. V. Nerkararyan, "Adiabatic nanofocusing of channel plasmon polaritons", Opt. Lett. 35, 541-543 (2010).

[17]. P. B. Johnson, R. W. Christy, “Optical constants of the noble metals”, Phys. Rev. B 6, 4370-4379 (1972).

[18]. M. Conforti, M. Guasoni, C. De Angelis, "Subwavelength diffraction management”, Opt. Lett. 33, 26622664 (2008).

[19]. S. A. Cummer, "An analysis of new and existing FDTD methods for isotropic cold plasma and a method for improving their accuracy", IEEE T. Antenn. Propag. 45, 392-400 (1997).

[20]. R. M. Joseph, T. Allen, "Spatial soliton deflection mechanism indicated by FD-TD Maxwell's equations modelling," IEEE Photonic. Tech. L. 6, 1251-1254 (1994).

\section{Introduction}

A plasmon is an optical mode resonant with the collective electronic oscillations of a conductor. The field dealing with the study of such modes is called plasmonics and is developing very fast at the moment as it may provide integrated devices with new properties and functionalities [1,2]. Including metals as part of optical elements will allow to overcome the diffraction limits due to the excitation of surface plasmons. As a consequence, light can be confined into subwavelength scales and photonic circuits will result compatible with nowadays electronics, in sizes as well as in fabrication techniques. Potential interesting applications of plasmonic devices are, to cite a few, colimators for highdensity data-store devices, high sensitivity sensors, high speed optical modulators and performance enhancement for solar cells.

One of the simplest plasmonic waveguides is an interface between a metal and a dielectric that supports the so-called surface plasmon polaritons. However, more complex systems as metal-dielectric-metal waveguides are interesting for a more efficient excitation of plasmonic modes and to keep optical losses low. In such guiding structures, light may be tightly confined between two metal slabs and it can even be used for efficient nanofocusing [3]. More complex systems as directional couplers, which are of crucial importance in integrated photonic circuits have also been studied theoretically and even demonstrated experimentally for different geometries [4-6]. This devices are based on cross-talk coupling between two neighboring waveguides and have been also suggested for converting the modes of an optical dielectric waveguide into plasmonic modes propagating along a thin metal stripe [7].

When nonlinear effects are taken into account, new functionalities for the mode control are provided. Nonlinear plasmonic waveguides were known for many years [8-10]. It was also shown that nonlinear slot waveguides created by a nonlinear dielectric slab sandwiched between two metals support subwavelength guided modes of different symmetries [11], including a novel type of asymmetric modes which is important for nonlinear switching. 
In this work we present a study of the mode structure and switching characteristics of two coupled nonlinear plasmonic slot waveguides with nonlinear dielectric cores surrounded by metallic layers [12]. The performance of those devices is strongly degraded due to the high power losses which take place because metals are not perfect conductors at optical frequencies. This is the main drawback of plasmonic devices and seeking solutions for avoiding the negative effect of losses is a priority in the research activities of this field. In this way we propose a modification of the device using tapered waveguides in order to rise the power density inside the cores and compensate for the power losses. Similar to isolated plasmonic waveguides [13-16], by an appropriate choice of the taper angle, we can effectively suppress the mode attenuation achieving an effective power transfer in both linear and nonlinear couplers.

\section{Nonlinear modes}

The Maxwell's equations for the electric, $\vec{\varepsilon}(\vec{R}, t)$, and magnetic, $\overrightarrow{\mathcal{H}}(\vec{R}, t)$, fields are:

$$
\begin{gathered}
\nabla \times \overrightarrow{\mathcal{E}}=-\mu_{0} \partial_{t} \overrightarrow{\mathcal{H}}, \\
\nabla \times \overrightarrow{\mathcal{H}}=\partial_{t} \overrightarrow{\mathcal{D}},
\end{gathered}
$$

where $\overrightarrow{\mathcal{D}}=\epsilon_{0} \in \overrightarrow{\mathcal{E}}$ is the electric displacement, $\mu_{0}$ and $\epsilon_{0}$ the vacuum permeability and permitivity respectively, and $\epsilon(\vec{R})$ the dielectric function describing the medium. We consider a planar coupler formed by two dielectric cores with a nonlinear Kerr response and separated a distance $d$, bounded by metallic linear claddings (see Fig. 1). We also consider harmonic fields of frequency $\omega$ and scale the spatial variables as $\vec{r}=k_{0} \vec{R}$, where $\vec{r} \equiv(x, y, z)$ and $k_{0}=\omega / c$, being $c$ the speed of light in vacuum. We assume the structure lying in the plane $X Z$ and take $z$ as the propagation direction. Besides, we consider TMpolarized fields (components $\mathcal{H}_{y}, \varepsilon_{x}$ and $\varepsilon_{z}$ ) and describe the medium by the dielectric function:

$$
\epsilon(\vec{r})=\left\{\begin{array}{l}
\epsilon_{m}, \text { for claddings } \\
\epsilon_{d}+\gamma\left(\left|\varepsilon_{x}\right|^{2}+\left|\varepsilon_{z}\right|^{2}\right), \text { for cores, }
\end{array}\right.
$$

where $\gamma$ accounts for the Kerr nonlinear response.

We seek stationary states of the form,

$$
\begin{aligned}
\overrightarrow{\mathcal{H}}(\vec{r}, t) & =\vec{H}(x) \frac{\exp [-i(\beta z-\omega t)]}{\mu_{0} c \sqrt{\gamma}}, \\
\vec{\varepsilon}(\vec{r}, t) & =\vec{E}(x) \frac{\exp [-i(\beta z-\omega t)]}{\sqrt{\gamma}},
\end{aligned}
$$

where $\beta$ is the propagation constant. Replacing Eqs. (3) in Eqs. (1) and considering TM-fields of the form $\vec{H}=H_{y} \hat{y}, \vec{E}=E_{x} \hat{x}+E_{z} \hat{z}$, we obtain the following $z$-independent system for the field components,

$$
\begin{gathered}
\partial_{x} H_{y}=-\epsilon E_{z}, \\
\partial_{x} E_{z}=\left(1-\frac{\beta^{2}}{\epsilon}\right) H_{y},
\end{gathered}
$$

together with the relationship $\epsilon E_{x}=\beta H_{y}$.

The system of ODEs is numerically solved by a relaxation method imposing the continuity of fields $H_{y}$ and $E_{z}$ at the boundaries between claddings and cores and a decaying behavior at $x= \pm \infty$. To carry out with the numerical calculations, we took $\epsilon_{d}=2.25, \epsilon_{m}=-8.25$ (silver for a wavelength $\lambda \approx 480 \mathrm{~nm}$ [17]), waveguide width $w=0.5$ and separation between waveguides $d=0.7$. The modes are characterized by the propagation constant $\beta$ and their power flux, given by the $z$-component of the Poynting vector, $P=\int(\vec{E} \times \vec{H}) \hat{z} d x=$ $\beta \int\left(H_{y}^{2} / \epsilon\right) d x$. Examples of the modes are presented in Fig. 1.
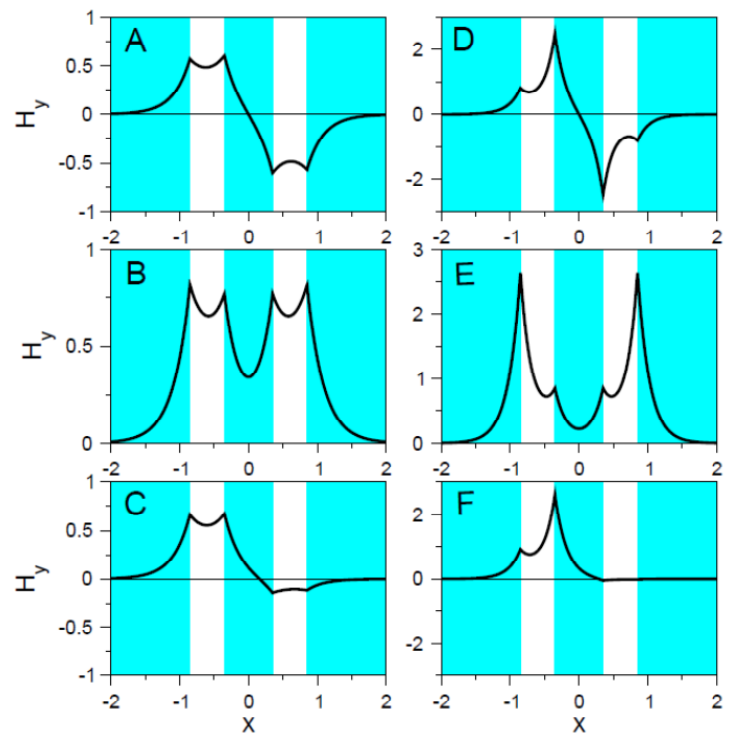

Fig. 1: Examples of the modes of the directional coupler for two different values of the propagation constant. A, B, C label the antisymmetric, symmetric and asymmetric modes for $\beta=$ 3. C, D, F label the corresponding modes for $\beta=5$. Labels correspond to the points marked in Fig. 2. 


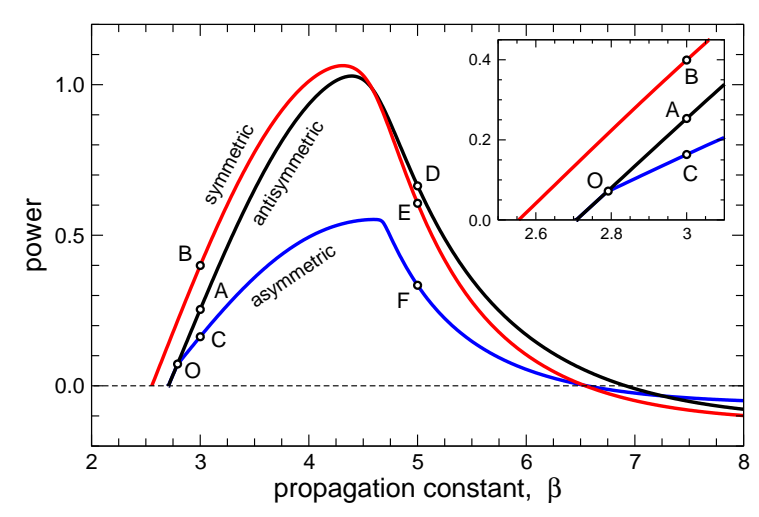

Fig. 2. Power curves versus propagation constant for the three different modes: antisymmetric, symmetric and asymmetric. Labels correspond to plots in Fig. 1.

Three different types of modes can be found: symmetric (A,D), antisymmetric (B,E), and asymmetric $(\mathrm{C}, \mathrm{F})$. In the linear limit the coupler supports both symmetric and antisymmetric modes $(A, B)[18]$, being the antisymmetric mode the fundamental, contrariwise as what happens in purely dielectric couplers. They are created by in-phase or out-of-phase symmetric modes of isolated slot waveguides. Above a power threshold a symmetry breaking takes place and a new asymmetric mode (C) emerges at the bifurcation point 0 (see Fig. 2). This mode is composed of two antisymmetric modes (since it bifurcates from a branch of antisymmetric solutions) but the filed amplitudes are different in the two slots. For larger powers, all those three modes evolve almost independently, and the field becomes mainly confined at the boundaries between dielectric and metallic layers in each waveguide. Such a high field concentration leads to the symmetry breaking in each of the slot waveguides, described earlier for an isolated nonlinear slot[11], so the amplitudes at the metal-dielectric surfaces become different (modes D and E). The field is shifted towards the metal cladding, so that the total power decreases, and it may even become negative due to the dominant (negative) contribution of the backward power flux concentrated in the metal (see Fig. 2).

\section{Switching properties}

We performed FDTD simulations to study the dynamics of the system. To account for the high dispersive metallic layers and make the algorithm numerically stable we use a classical model for cold plasmas that we introduced in Eqs. (1) writing the displacement vector as $\overrightarrow{\mathcal{D}}=\epsilon_{0} \vec{\varepsilon}+\partial_{t} \overrightarrow{\mathcal{P}}$ and modelling the polarization current $\overrightarrow{\mathcal{J}}=\partial_{t} \overrightarrow{\mathcal{P}}$ by the equation[19]:

$$
\partial_{t} \overrightarrow{\mathcal{P}}+\Gamma \overrightarrow{\mathcal{P}}=\epsilon_{0} \omega_{p}^{2} \vec{\varepsilon},
$$

where $\omega_{p}$ is the plasma frequency and $\Gamma$ is the electron collision frequency that accounts for the power losses. Both parameters can be calculated considering the Drude model $\epsilon(\omega)=1$ $\omega_{p}^{2} /\left(\omega^{2}+i \Gamma \omega\right)$ and using the values $\operatorname{Re}(\epsilon)=-8.25$ and $\operatorname{Im}(\epsilon)=0.3$, deduced from the optical constants of silver at $\lambda \approx 480 \mathrm{~nm}$ [17]. Taking $\omega=1$, i.e. time is measured in the units of $\omega^{-1}$, we obtain $\omega_{p}=3.043$ and $\Gamma=0.032$. On the other hand, to model the dielectric cores, we consider an instantaneous nonlinear response [20] and use Eqs. (2) and (1). A single Newton step is used to obtain the electric field components from the resulting cubic equation. This step guarantees the suppression of numerical instabilities while keeping the efficiency of the calculation. On the other hand, we implemented perfect matching layers (PML) for all the boundary conditions of the calculation domain.

We initially neglected losses for simplicity. In Fig. 3, top, we present three images correspondent to simulations carried out for different input power to illustrate how power flux can be switched from the second core to the first one just controlling input power. In other to get quantitative results power is obtained from the simulations integrating the Poynting vector in the transversal variable $x$ at $z=z_{b}$ where $z_{b}$ is the beat length, i.e. the distance at which power completely transfers to the second core under linear propagation. This is done after propagating for the necessary number of timesteps and performing further steps to time average the Poynting vector. In Fig. 4 we show the switching curve $(\Gamma=0)$ where the normalized output power $P_{0} / P$ is plotted against the input power $P_{0}$, showing how power transfer to the second core is suppressed when power raises over a threshold. On the other hand, when realistic losses are taken into account, switching is still possible as shown in Fig. 3, bottom, where 

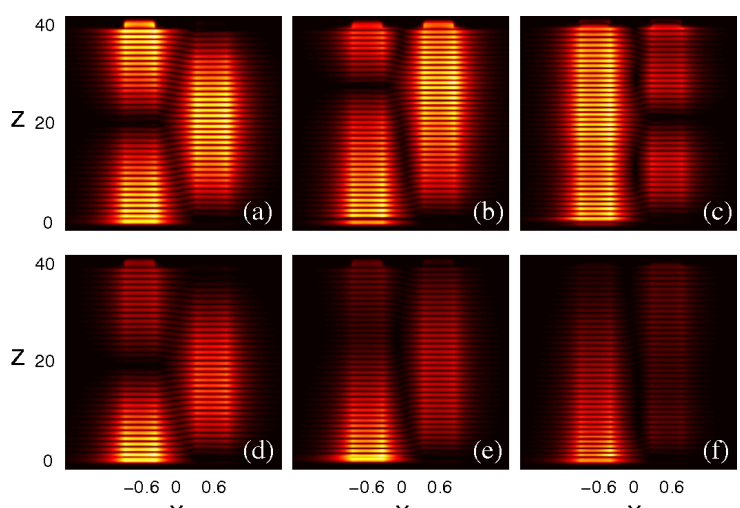

$\mathrm{x}$

$\mathrm{X}$

Fig. 3. Images illustrating switching with the plasmonic coupler. Top row: cases of linear (a), intermediate power (b) and nonlinear (c) propagation without taking into account losses. Bottom row: linear (d), intermediate (e) and nonlinear (f) regimes when realistic losses are considered. It is represented the modulus of the magnetic component.

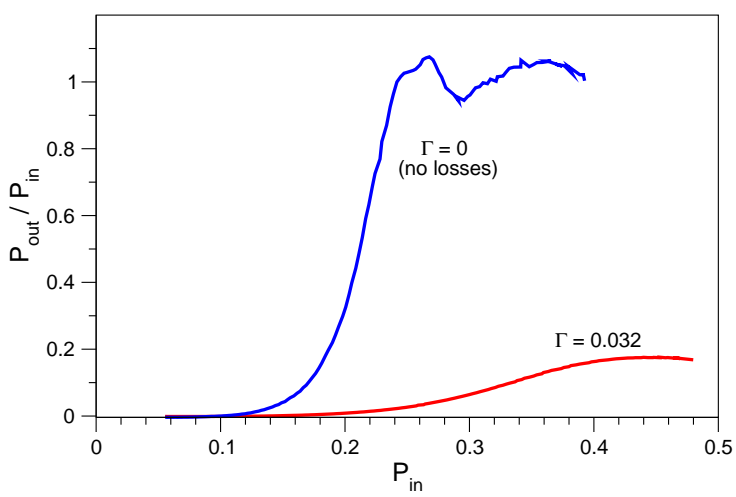

Fig. 4. Switching curves for the plasmonic coupler with losses neglegted $(\Gamma=0)$ and for realistic losses. The plot shows output power after a beat length (normalised to the input power) versus input power.

the three corresponding simulations for quasilinear, intermediate and nonlinear regime are displayed. In Fig. 4 we also plot the switching curve for this case, showing the expected decrease of power and a large decreasing of the curve slope. This means that switching does not take place any more for a sharp power threshold and the efficiency of the device is strongly decreased.

\section{Tapered couplers}

In order to improve the efficiency of the coupler when realistic losses are considered, we have also studied the switching properties when the waveguides are designed with a slightly tapered

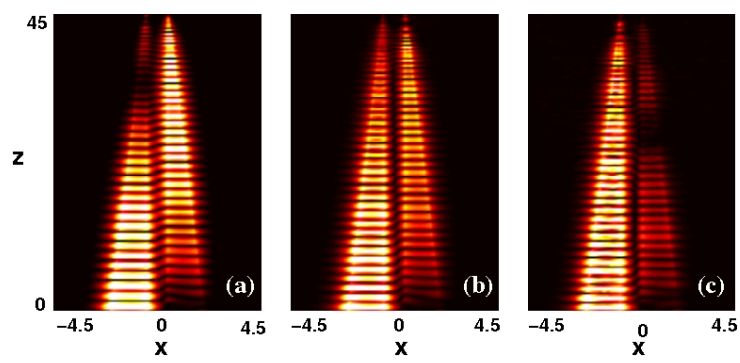

Fig. 5. Linear (a), intermediate (b) and nonlinear (c) power regimes for the coupler with tapered waveguides, taking into account realistic losses. The modulus of the magnetic component is shown.

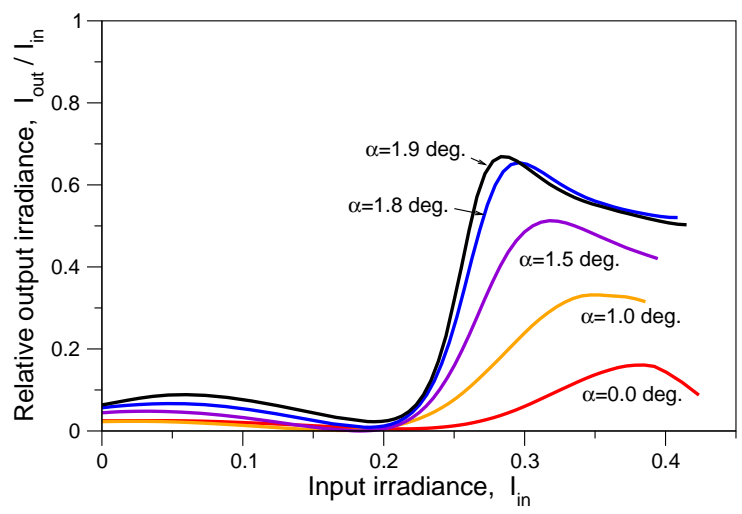

Fig. 6. Switching curves for the tapered-waveguide coupler for different taper angles $(\alpha)$. The plot shows output irradiance after a beat length (normalised to the input irradiance) versus input irradiance.

shape. This allows to induce a power concentration inside the cores that compensates for losses. FDTD simulations were carried out for different values of the tapering angle. In Fig. 5 we show images representing the modulus of the field (magnetic component) simulated for nonlinear cores and different power regimes for tapered waveguides at an angle of $\alpha=1.9 \mathrm{deg}$. The three images correspond to linear, intermediate and nonlinear regimes reached when input power is raised. Though losses do still exist, the irradiance or power density inside the cores is enhanced and this is the reason why switching results more effective. This is clearly seen in the switching curves shown in Fig. 6 where the relative irradiance for a beat length is plotted against the input irradiance, for different values of the taper angle. For increasing angles the output irradiance is also increased as well as the slope of the switching curves making the switching threshold increasingly sharper. 


\section{Conclusions}

We have studied nonlinear modes and switching properties of planar directional couplers created by two coupled slot metal-dielectric-metal waveguides. We have discussed nonlinear switching and evaluating the effect of power losses on the performance of the device. We have also proposed the use of tapered waveguides to compensate for losses. The results of the simulations show that the efficiency is strongly increased when the taper angle is properly selected.

\section{Acknowledgements}

We thank the Australian Research Council, Ministerio de Ciencia e Innovación of Spain (project MAT2008-06870) and Xunta de Galicia (project 10PXIB265118PR) for supporting this work. 